\title{
Um modelo matemático de câncer com quimioterapia e imunoterapia
}

\author{
Nilo A. Martin, \\ Braile Biomédica, \\ 15091-450, São José do Rio Preto, SP \\ E-mail: nilomartin@terra.com.br \\ Gustavo Cruz-Pacheco \\ IIMAS, UNAM \\ Cidade do México, México \\ E-mail: gcruzpacheco@gmail.com \\ Paulo F. A. Mancera \\ Departamento de Bioestatística, IBB, Unesp \\ 18618-970, Botucatu, SP \\ E-mail: pmancera@ibb.unesp.br
}

\begin{abstract}
Resumo: O câncer surge a partir de mutações, causadas no DNA celular, que resultam na multiplicação descontrolada destas células, dando origem a uma massa tumoral que pode comprometer o funcionamento de órgãos e tecidos, e, consequentemente, do organismo como um todo. A importância da busca por novas abordagens de tratamentos e prevenção do câncer é evidente, perante aos indicadores de incidência e de óbitos causados pela doença. As principais modalidades de tratamento ao câncer atualmente, como a quimioterapia, radioterapia e cirurgia, nem sempre se mostram eficientes e, além disso, apresentam pesados efeitos colaterais, comprometendo a qualidade de vida dos pacientes submetidos a eles. Emerge deste cenário uma modalidade de tratamento voltada a estimular o sistema imunológico no combate ao câncer, denominada imunoterapia. Neste trabalho buscamos, através de um modelo de equações diferenciais ordinárias, analisar a influência de tratamentos que utilizam componentes do sistema imunológico sobre crescimento tumoral, comparando seus efeitos separadamente e em conjunto com diferentes protocolos de tratamento quimioterápico. A partir de simulações numéricas realizadas, obtivemos indicativos de que, em alguns casos, dependendo da estratégia de tratamento e do estágio do desenvolvimento tumoral no qual esta é aplicada, a doença pode ser contida, possibilitando uma sobrevida pós-tratamento prolongada.
\end{abstract}

Palavras-chave: Quimioterapia, Imunoterapia, Câncer

\section{Modelo matemático}

A principal função do sistema imunológico é evitar que agentes patogênicos (vírus, bactérias e parasitas) se desenvolvam no organismo, agindo como um sistema de constante vigilância. Os agentes patogênicos são reconhecidos pelo sistema imunológico através de proteínas expressas em suas superfícies, chamadas de antígenos, responsáveis por desencadear a resposta imunológica do organismo. Da mesma maneira, células cancerígenas também expressam antígenos em sua superfície e podem desencadear uma resposta imunológica, porém, diferentemente de agentes patogênicos, as células cancerígenas se originam de células normais, e expressam proteínas muito semelhantes às expressas pelas células normais e, então, o reconhecimento dos seus antígenos 
pelo sistema imunológico pode não ser completamente eficiente. Através do estudo da interação do sistema imunológico com células cancerígenas, procura-se maneiras de aprimorar a ação do sistema imunológico sobre o crescimento tumoral, estimulando o reconhecimento do sistema imunológico aos antígenos expressos pelas células cancerígenas. Os avanços científicos neste campo trazem reais aplicações do conhecimento da imunologia ao campo da oncologia na forma de imunoterapias e vacinas terapêuticas. A utilização de componentes do sistema imunológico no combate ao câncer ainda não é utilizada amplamente em tratamentos clínicos, pois muitos aspectos desta interação permanecem desconhecidos. Apesar de haver um conhecimento consistente sobre as respostas imunológicas aos mais diversos agentes patogênicos, esta relação com o câncer se mantém, ao menos em partes, incompreendida. Diferentes fatores contribuem para a dificuldade do entendimento da "relação" entre o sistema imunológico e o crescimento tumoral, e destas, algumas relacionam-se à elevada instabilidade genética das células neoplásicas. Assim sendo, com base em [4] e [5], propomos um modelo matemático que envolve a influência do sistema imunológico sobre o crescimento tumoral, com o objetivo de ilustrar algumas possíveis situações resultantes dessa interação, considerando alguns cenários que levam em conta condições que refletem aspectos observados na realidade. Iremos considerar também a dinâmica angiogênica e a inserção de um tratamento quimioterápico no modelo, bem como, a presença de células normais. A equação que modela a presença de células do sistema imunológico baseia-se em [2] e [4], e engloba diferentes aspectos da resposta imunológica mediados por diferentes tipos de células, como o estímulo da ação de células efetoras e o ataque às células neoplásicas, ou seja, esta representa a variação do número de células do sistema imunológico como um todo, não havendo distinção entre os diferentes tipos celulares. Quanto à variação de células endoteliais no sistema, nos baseamos em [1] e [5]. A equação que modela esta dinâmica leva em conta a liberação de fatores estimulantes e inibitórios à vascularização pelas células tumorais, a taxa de reprodução das células endoteliais e o efeito da quimioterapia sobre tais células, agregando ao modelo efeitos de protocolos antiangiogênicos de quimioterapia. Com relação à quimioterapia, iremos considerar a ação de uma única droga, pois, apesar de serem, usualmente, utilizadas combinações de diferentes drogas quimioterápicas em algumas modalidades de tratamento, seus efeitos variam de acordo com as drogas em questão e o tipo de câncer, sendo as respostas nem sempre aditivas. Além disso, levaremos em conta o tratamendo quimioterápico neo-adjuvante, pois aqui a quimioterapia será o principal tratamento considerado.

Sendo $N_{1}(t)$ o número de células tumorais, $N_{2}(t)$ o número de células normais, $I(t)$ o número de células do sistema imunológico, $L(t)$ o número de células endoteliais e $Q(t)$ a quantidade de quimioterápico no sistema em função do tempo, o modelo proposto tem a seguinte forma:

$$
\left\{\begin{array}{l}
\frac{d N_{1}}{d t}=r_{1} N_{1}\left(1-\frac{N_{1}}{k_{1}+L}-\frac{\alpha_{12} N_{2}}{k_{1}+L}\right)-c_{1} I N_{1}-\frac{\mu N_{1} Q}{a+Q} \\
\frac{d N_{2}}{d t}=r_{2} N_{2}\left(1-\frac{N_{2}}{k_{2}}-\frac{\alpha_{21} N_{1}}{k_{2}}\right)-\frac{\nu N_{2} Q}{b+Q} \\
\frac{d I}{d t}=s-d_{1} I+\frac{\rho I N_{1}}{\gamma+N_{1}}-c_{2} I N_{1}-\frac{\delta I Q}{d+Q} \\
\frac{d L}{d t}=\sigma L+\phi N_{1}-\omega N_{1} L-\frac{\eta L Q}{c+Q} \\
\frac{d Q}{d t}=q-\lambda Q
\end{array}\right.
$$

em que todas as constantes, descritas a seguir, e condições iniciais $N_{1}(0), N_{2}(0), I(0), L(0)$ e $Q(0)$ são maiores do que zero. Definimos a capacidade suporte da população de células tumorais dependente da quantidade de células endoteliais no sistema, dada por $k_{1}+L$, e $k_{2}$ sendo a capacidade suporte para as células normais; $\alpha_{i j}$, o coeficiente de competição entre as populações, 
mensura os efeitos da população $j$ na população $i ; r_{i}(i=1,2)$ são as respectivas taxas de crescimento de cada população; os parâmetros $\rho$ e $\gamma$ estão relacionados à antigenicidade das células tumorais em questão, pois tais parâmetros ditam a velocidade da reação; $d_{1}$ representa a taxa de mortalidade das células imunológicas quando não há células tumorais; $-c_{1} I N_{1} \mathrm{e}-c_{2} I N_{1}$, respectivamente, diz respeito à morte das células tumorais pela ação do sistema imunológico e à inativação das células imunológicas quando agindo nas células tumorais. O termo $s$ refere-se a uma fonte de células imunológicas externa ao sistema, e, então, se $s>0$ podemos modelar a inserção no sistema de agentes imunológicos através, por exemplo, de uma vacina, e, então, interpretamos este parâmetro como um termo de tratamento a partir da imunização passiva, enquanto que o termo $\rho$, associado à intensidade da resposta imunológica devido à presença do tumor, é associado à imunização ativa. Com relação à variação do número de células entoteliais no sistema, $L(t)$, definimos $\phi N_{1}$ e $\omega N_{1} L$ em representam, respectivamente, a liberação de fatores estimulantes de crescimento das células endoteliais pelo tumor (VEGF, Vascular Endothelial Growth Factor), e os fatores de inibição de crescimento das células endoteliais (TSP-1), que são secretados mediante a presença dessas células na região tumoral e proporcionalmente ao número de células que compõem a massa tumoral. Para finalizar $\sigma$ representa a taxa de reprodução das células endoteliais; $\mu$ e $\nu$ são as taxas de mortalidade das células tumoral e normal devido a droga, respectivamente; $\lambda$ é a taxa de decaimento do quimioterápico; $\phi$ está relacionado com a liberação de fatores de crescimento; $\omega$ modela a inibição da vascularização provocada pelo próprio tumor; $a, b, c$ e $d$ podem ser interpretados como determinantes para a velocidade da reação de cada população celular à droga quimioterápica.

A função $q(t)$ assume duas formas diferentes, de acordo com o tipo de estudo a ser feito:

1. Para estudarmos o modelo analiticamente, assumiremos $q(t)>0$, e constante.

2. Para as simulações numéricas, iremos considerar a função $q(t)$ periódica e definida de acordo com [3] da seguinte forma:

$$
q(t)=\left\{\begin{array}{cc}
q>0, & n<t \leq n+\tau \\
0, & n+\tau<t \leq n+T
\end{array},\right.
$$

em que $T$ é consiste no tempo entre as infusões da droga, $n=0, T, 2 T, 3 T, \ldots$ e $\tau$ consiste no tempo de infusão, $\operatorname{com} T>\tau$.

\section{Resultados e discussões}

Foram estudados diferentes cenários, nos quais foram simuladas diferentes combinações de estratégias de tratamento. Além disso, realizamos o estudo da estabilidade do sistema, com o objetivo de encontrar condições sob as quais seria observada a eliminação das células tumorais. Através da análise de estabilidade do sistema, obtivemos os seguintes pontos de equilíbrio:

- $S_{1}=\left(N_{1}, N_{2}, I, L, Q\right)=\left(0,0, \frac{s(q+d \lambda)}{q \delta+q d_{1}+d \lambda d_{1}}, 0, \frac{q}{\lambda}\right)$.

- $S_{2}=\left(N_{1}, N_{2}, I, L, Q\right)=\left(0, \frac{q \nu k_{2}+q k_{2} r_{2}+b \lambda k_{2} r_{2}}{(q+b \lambda) r_{2}}, \frac{s(q+d \lambda)}{q \delta+q d_{1}+d \lambda d_{1}}, 0, \frac{q}{\lambda}\right)$.

- $S_{3}=\left(N_{1}, N_{2}, I, L, Q\right)=\left(\overline{N_{1}}, 0, \bar{I}, \bar{L}, \bar{Q}\right)$.

- $S_{4}=\left(N_{1}, N_{2}, I, L, Q\right)=\left(N_{1}^{*}, N_{2}{ }^{*}, I^{*}, L^{*}, Q^{*}\right)$.

Obtemos analiticamente apenas os autovalores $S_{1}$ e $S_{2}$. A partir daí, concluímos que, para o caso da erradicação das células tumorais, não é possível que o ponto $S_{2}$ seja estável, independente da estratégia de tratamento, devido á vascularização tumoral. Observamos que as situações $S_{1}$ 
e $S_{3}$ não são viáveis em termos biológicos, e que o efeito da angiogênese é modelado apenas nas células tumorais, o que implica em $N_{1}=L=0$ em $S_{2}$.

Partimos então para as simulaçẽs numéricas, a fim de observar as condições de tratamento sob as quais a doença pode ser contida. Para tanto, simulamos situações nas quais foram considerados tratamentos com imunoterapia ativa e passiva, e a combinação destas modalidades de tratamento com a quimioterapia, infundida sob o protocolo MTD e metronômico.

Tabela 1: Parâmetros utilizados nas simulações numéricas do modelo.

\begin{tabular}{cccc}
\hline Parâmetro & Valor & Unidade & Referência \\
\hline$r_{1}$ & $10^{-2}$ & dia $^{-1}$ & Rodrigues, 2011 \\
$\alpha_{12}$ & $9 \times 10^{-5}$ & - & Rodrigues, 2011 \\
$k_{1}$ & $10^{7}$ & célula $^{-11}$ & Rodrigues, 2011 \\
$c_{1}$ & $1 \times 10^{-11}$ & célula dia $^{-1}$ & Kuznetsov, 1994 \\
$\mu$ & 1.3 & $\mathrm{mg}^{-1}$ & valor assumido \\
$a$ & $2 \times 10^{3}$ & $\mathrm{mg}$ & Rodrigues, 2011 \\
$r_{2}$ & $10^{-3}$ & $\mathrm{dia}^{-1}$ & $r_{2}<r_{1}$ \\
$\alpha_{21}$ & $9 \times 10^{-2}$ & - & Rodrigues, 2011 \\
$k_{2}$ & $10^{12}$ & célula $^{2}$ & valor assumido \\
$\nu$ & 0.08 & $\mathrm{mg}^{-1}$ & $\nu \ll \mu$ \\
$b$ & $5 \times 10^{6}$ & $\mathrm{mg}^{-1}$ & Rodrigues, 2011 \\
$d_{1}$ & $2 \times 10^{-1}$ & $\mathrm{dia}^{-1}$ & de Pillis, 2001 \\
$\gamma$ & $1 \times 10^{2}$ & célula $^{-12}$ & de Pillis, 2001 \\
$c_{2}$ & $1 \times 10^{-12}$ & células dia $^{-1}$ & Kuznetsov, 1994 \\
$\delta$ & $1 \times 10^{-1}$ & $\mathrm{dia}^{-1}$ & valor assumido \\
$d$ & $5 \times 10^{6}$ & $\mathrm{mg}^{-3}$ & $d \sim b$ \\
$\sigma$ & $1 \times 10^{-3}$ & $\mathrm{dia}^{-1}$ & $\sigma \sim r_{2}$ \\
$\phi$ & 1 & $\mathrm{dia}^{-1}$ & valor assumido \\
$\omega$ & $1 \times 10^{-12}$ & $\mathrm{dia}^{-1}$ & Rodrigues, 2011 \\
$\eta$ & 1 & $\mathrm{mg}^{-1}$ & Rodrigues, 2011 \\
$c$ & $2 \times 10^{3}$ & $\mathrm{mg}^{-1}$ & Rodrigues, 2011 \\
$\lambda$ & 4.16 & $\mathrm{dia}^{-1}$ & Rodrigues, 2011 \\
\hline
\end{tabular}

Na Figura 1, é ilustrada a dinâmica do modelo sob a influência do tratamento com imunização ativa. Notamos que ocorre uma resposta imunológica intensa devido à presença das células tumorais, entretanto tal resposta não é eficaz a ponto de controlar o crescimento tumoral, o que resulta em osculações em ambas as populações, com a população de células tumorais atingindo tamanhos máximos de aproximadamente $10^{9}$ células.

Por outro lado, quando consideramos o tratamento com a imunoterapia passiva, observamos uma ação mais eficiente do sistema imunológico sobre as células tumorais, que resulta na estabilização na população de células tumorais em uma quantidade praticamente inofensiva, possibilitando uma maior sobrevida para o indivíduo. Tal situação é ilustrada na Figura 2.

Buscando obter uma maior eficiência nos tratamentos, adicionamos a cada uma das situações ilustradas anteriormente os efeitos da quimioterapia. Exibimos aqui, apenas a combinação das duas modalidades de imunoterapia com a quimioterapia infundida sob o protocolo metronômico, pois este, a princípio, se mostrou mais eficiente.

Podemos notar que, mesmo quando sob a ação de ambos os tratamentos, não obtivemos a erradicação das células tumorais. Entretanto, quando combinamos a imunoterapia passiva com a quimioterapia, situação ilustrada na Figura 4, o crescimento tumoral é contido em pouco 


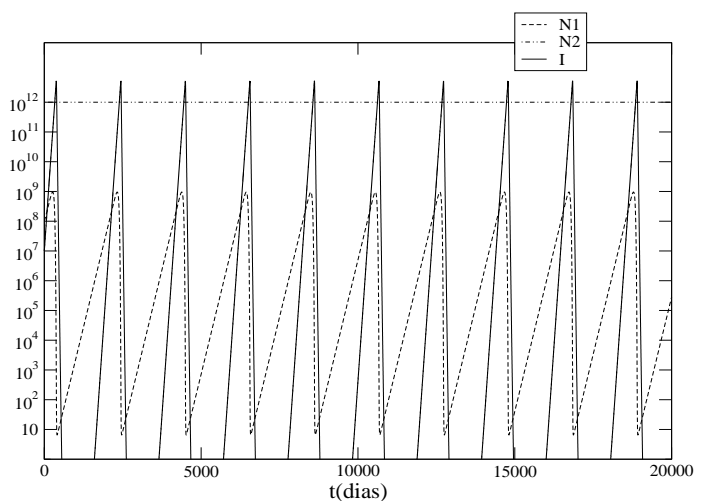

Figura 1: Simulação numérica do sistema considerando o tratamento com imunoterapia ativa, com

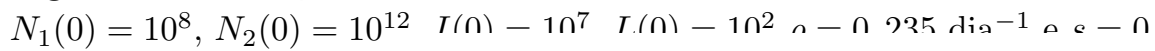

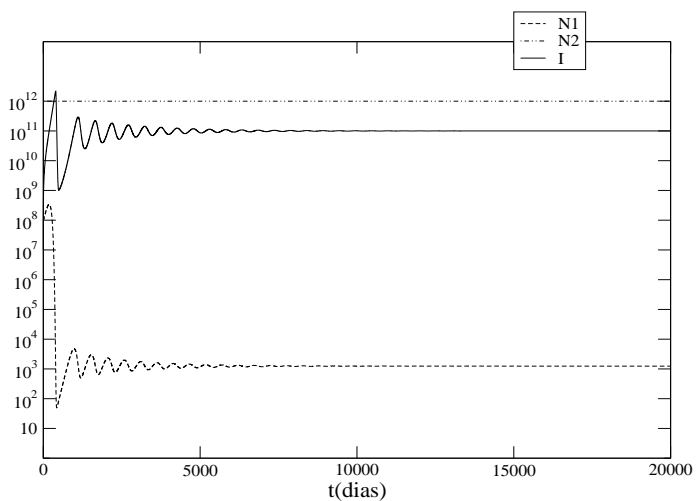

Figura 2: Simulação numérica do sistema considerando o tratamento com imunoterapia passiva, com $N_{1}(0)=10^{8}, N_{2}(0)=10^{12}, I(0)=10^{7}, L(0)=10^{2} \rho=0,215 \operatorname{dia}^{-1}$ e $s=10^{8}$

tempo, se estabilizando em uma quantidade de aproximadamente $10^{2}$ células, o que representaria uma massa tumoral inofensiva ao ser humano caso continuasse neste tamanho. Tal situação nos remete a ideia de que, em alguns casos, a busca pela contenção e pelo controle do crescimento tumoral é tão importante quanto a busca pela erradicação total do mesmo.

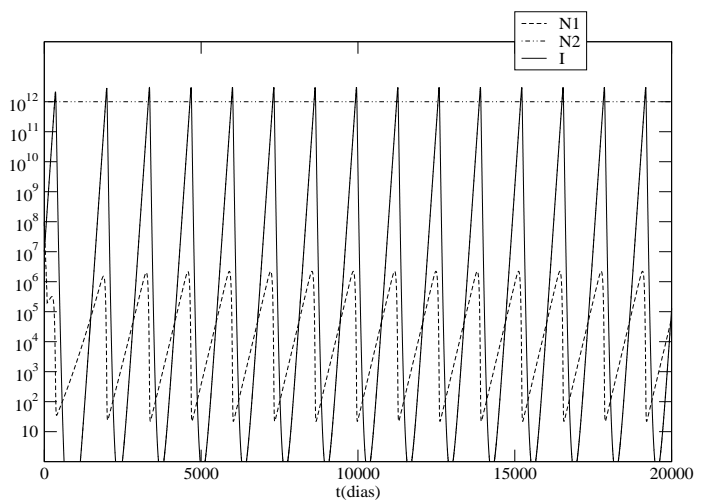

Figura 3: Simulação numérica do sistema considerando o tratamento com imunoterapia ativa, com $N_{1}(0)=10^{8}, N_{2}(0)=10^{12}, I(0)=10^{7}, L(0)=10^{2} \rho=0,235 \mathrm{dia}^{-1}$ e $s=0$, em conjunto com a quimioterapia, infundida sob o protocolo metronômico. 


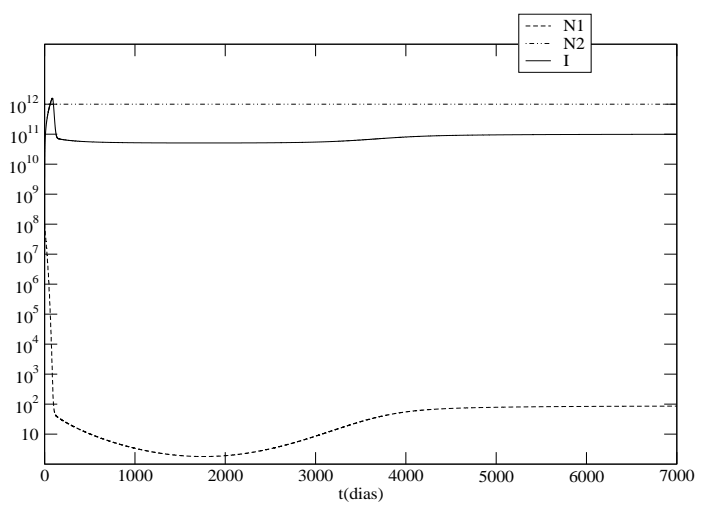

Figura 4: Simulação numérica do sistema considerando o tratamento com imunoterapia passiva, com $N_{1}(0)=10^{8}, N_{2}(0)=10^{12}, I(0)=10^{7}, L(0)=10^{2} \rho=0,215 \mathrm{dia}^{-1}$ e $s=10^{10}$, em conjunto com a quimioterapia, infundida sob o protocolo metronômico.

\section{Conclusões}

Quando consideramos a infusão da quimioterapia através do protocolo metronômico, que apresenta um efeito sobre o processo de angiogênese, em conjunto com a imunoterapia, tal estratégia de tratamento se mostrou mais eficiente em conter o crescimento tumoral, quando comparada à infusão segundo o protocolo MTD. Além disso, notamos que, dentre as modalidades de imunoterapia mencionadas, a imunoterapia passiva se mostrou mais eficaz em agir sobre as células cancerígenas.

\section{Referências}

[1] P. Hahnfeldt, D. Panigrahy, J. Folkman, L. Hlatky, Tumor development under angiogenic signaling: a dynamical theory of tumor growth, treatment response, and postvascular dormancy, Cancer Res, 59 (1999) 4770-4775.

[2] V. A. Kuznetsov, I. A. Makalkin, Nonlinear dynamics of immunogenic tumors: parameter estimation and global bifurcation analysis, Bull Math Biol, 56(2) (1994) 295-321.

[3] R. Martin, K. L. Teo, "Optimal control of drug administration in cancer chemotherapy", World Scientific, Singapore, 1993.

[4] L. G. de Pillis, A. Radunskaya, A mathematical tumor model with immune resistance and drug therapy: an optimal control approach, J Theor Med, 3 (2001) 79-100.

[5] D. S. Rodrigues, "Modelagem matemática em câncer: dinâmica angiogênica e quimioterapia antineoplásica", Dissertação de Mestrado, Biometria-Unesp, 2011. 